\title{
Effects of interfacial polymerization conditions on performance of polyamide reverse osmosis membranes and optimization of polymerization conditions by statistical methodology
}

\author{
Hyung-Gyu Park ${ }^{\mathrm{a}}$, Yong-Doo Jung ${ }^{\mathrm{b}}$, Sung-Pyo Hong ${ }^{\mathrm{c}}$, Young-Nam Kwon, ${ }^{\mathrm{a}, *}$ \\ ${ }^{a}$ School of Urban and Environmental Engineering, Ulsan National Institute of Science and Technology (UNIST), 100 Banyeon-ri, \\ Ulsan 689, Korea, emails: kwonyn@unist.ac.kr(Y.-N. Kwon), hkpark@unist.ac.kr (H.-G. Park) \\ ${ }^{b}$ Department of Biosystems and Biomaterials Science and Engineering, Seoul National University, 599 Gwanangno, Gwanak-gu, \\ Seoul 151-921, Korea, email: yong2@snu.ac.kr \\ 'RO Development Team, Toray Chemical Korea, 287, Gongdan-Dong, Gumi, Kyungbuk 730-707, Korea, email: alex.hong@toray-tck.com
}

Received 8 August 2016; Accepted 21 February 2017

\begin{abstract}
A B S T R A C T
Effect of fabrication parameters on the performance of polyamide reverse osmosis membrane was systematically investigated and the design of experiment (DOE) method was used as a tool for assessing the degree of contribution of individual parameters on the resultant membrane performance; this was in order to determine the optimized combination of the various parameters. The parametric studies for fabrication included reaction time, temperature of monomer containing solutions, type of organic solvents and monomer concentration. Reaction time controlled the amount of $m$-phenylenediamine (MPD) diffusing into organic solvents, and the temperature of MPD solution determined the hydrolysis rate of trimesoyl chloride (TMC) taking place exclusively in aqueous phase. Seven organic solvents were investigated to generate various diffusion rate of MPD monomers into the organic solvents, and the thin-film composite (TFC) membranes prepared from higher diffusion rate of MPD into organic solvents showed dense and thinner polyamide layers. Concentration of TMC in organic phase and MPD in aqueous phase showed the effects on the amount of TMC available on the reaction zone and the degree of MPD diffusing across the interface, respectively. Optimum combination of MPD, TMC and aqueous additive concentrations was established with the DOE method for TFC polyamide reverse osmosis (RO) membrane. The reliability of optimum settings for $\mathrm{RO}$ membrane preparation condition was verified by confirmation experiments. This study showed that various combinations of monomer concentration for fabrication of $\mathrm{RO}$ membranes with tailor-made performance could be provided from DOE and response surface methodology analysis.
\end{abstract}

Keywords: Polyamide; Reverse osmosis membrane; Interfacial polymerization; Design of experiment

\footnotetext{
* Corresponding author.
} 